\title{
Aligning Text and Phonemes for Speech Technology Applications Using an EM-Like Algorithm
}

\author{
R.I. DAMPER* \\ Image, Speech and Intelligent Systems (ISIS) Research Group, School of Electronics and Computer Science, \\ University of Southampton, Southampton SO17 1BJ, UK \\ rid@ecs.soton.ac.uk \\ Y. MARCHAND \\ Institute for Biodiagnostics (Atlantic), National Research Council Canada, Neuroimaging Research Laboratory, \\ 1796 Summer Street, Suite 3900, Halifax, Nova Scotia, Canada B3H 3 A7 \\ J.-D. S. MARSTERS AND A.I. BAZIN \\ Image, Speech and Intelligent Systems (ISIS) Research Group, School of Electronics and Computer Science, \\ University of Southampton, Southampton SO17 1BJ, UK
}

\begin{abstract}
A common requirement in speech technology is to align two different symbolic representations of the same linguistic 'message'. For instance, we often need to align letters of words listed in a dictionary with the corresponding phonemes specifying their pronunciation. As dictionaries become ever bigger, manual alignment becomes less and less tenable yet automatic alignment is a hard problem for a language like English. In this paper, we describe the use of a form of the expectation-maximization (EM) algorithm to learn alignments of English text and phonemes, starting from a variety of initializations. We use the British English Example Pronunciation (BEEP) dictionary of almost 200,000 words in this work. The quality of alignment is difficult to determine quantitatively since no 'gold standard' correct alignment exists. We evaluate the success of our algorithm indirectly from the performance of a pronunciation by analogy system using the aligned dictionary data as a knowledge base for inferring pronunciations. We find excellent performance - the best so far reported in the literature. There is very little dependence on the start point for alignment, indicating that the EM search space is strongly convex. Since the aligned BEEP dictionary is a potentially valuable resource, it is made freely available for research use.
\end{abstract}

Keywords: text-to-speech synthesis, string alignment, dynamic programming, EM algorithm, pronunciation by analogy

\section{1. Introduction}

28 The requirement commonly arises in speech technol-

29 ogy and natural language processing to align two lin-

30 ear, symbolic representations of the same linguistic en-

31 tity. One important example, which forms the focus of

32 this paper, is the alignment of the textual (orthographic

*To whom all correspondence should be addressed. or spelling) and phonemic (pronunciation) representations of isolated words (of English, in this work). The necessity to align text and phonemes arises in, for instance, inferring the complete form of spellingpronunciation word pairs from elliptical entries in a dictionary (Lawrence and Kaye, 1986) and adding new entries to the pronunciation dictionary that provides a mapping between sub-word models and language models in automatic speech recognition (Knill and Young, 
1997, p. 48). But as (Jansche, 2001) writes: "The problem of finding a good alignment has not received its due attention in the literature".

Two examples from the domain of text-tospeech (TTS) synthesis suffice to motivate the search for powerful automatic alignment techniques.

1. In (supervised) training of neural networks to perform spelling-to-sound conversion, as in the wellknown NETtalk and NETspeak of Sejnowski and Rosenberg (1987) and McCulloch et al. (1987) respectively, it is necessary to associate each letter of an input word with a target output phoneme. In both works, alignment was done manually, but this is time-consuming, error-prone, and limits the size of datasets that can be used for training. As speech synthesis becomes ever more data-driven (Damper, 2001) using ever larger dictionaries and corpora (Young and Bloothooft, 1997), so manual alignment becomes less and less tenable and the need for automatic alignment methods increases.

2. Increasingly in recent years, an approach known as pronunciation by analogy $(\mathrm{PbA})$ has been used in TTS synthesis to derive pronunciations for unknown words, i.e., those not listed in the system dictionary (Dedina and Nusbaum, 1991; Sullivan and Damper, 1993; Pirrelli and Federici, 1994; Pirrelli and Federici, 1995; Federici et al., 1995; Damper and Eastmond, 1996; Yvon, 1996a; Yvon, 1996b; Damper and Eastmond, 1997; Bagshaw, 1998; Damper et al., 1999; Pirrelli and Yvon, 1999; Marchand and Damper, 2000; Sullivan, 2001). $\mathrm{PbA}$ assembles pronunciations for such (unknown) words from partial matches to the (known) words listed in the dictionary-a process that requires each letter of every word in the dictionary to be aligned with a corresponding phoneme in contiguous, one-to-one fashion.

However, automatic alignment is a difficult problem. Much of the difficulty arises because of the lack of regularity ('consistency' and 'transparency') in the English writing system. By 'consistency', we mean that the same letter always corresponds to the same phoneme. In fact, English is notorious for the lack of consistency in its spelling-to-sound correspondence (Venezky, 1965; Carney, 1994) at the level of single letters. For instance, the letter $c$ is pronounced /s/ in cider but $/ \mathrm{k} /$ in cat. On the other hand, the $/ \mathrm{k} /$ sound of kitten is written with a letter $k$. By 'transparency', we mean that a single letter corresponds to a single phoneme (Henderson, 1984, p. 17) and vice versa.

The lack of consistency in English orthography is problematic for alignment since any given letter can potentially align with (i.e., correspond to) many different phonemes. To illustrate the problems that arise from lack of transparency, consider the word (quay, /ki/), for which a reasonable alignment might be:

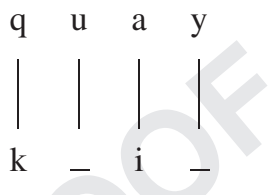

This word is not unusual for English in having fewer 99 phonemes than letters, necessitating the insertion of $\mathbf{1 0 0}$ 'null phonemes' in the transcription if a one-to-one $\mathbf{1 0 1}$ mapping is to be maintained. Such null symbols are $\mathbf{1 0 2}$ entirely 'artificial' in that they play no role in speci- $\mathbf{1 0 3}$ fying the pronunciation; their only purpose is to main- 104 tain the one-to-one correspondence between letters and $\mathbf{1 0 5}$ phonemes. Yet it is not clear precisely where the null $\mathbf{1 0 6}$ letters should be placed, since the following is also a $\mathbf{1 0 7}$ reasonable alignment:

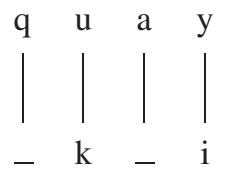

109

This example illustrates a key aspect of the lack $\mathbf{1 1 0}$ of transparency in that letter combinations frequently $\mathbf{1 1 1}$ correspond to a single phoneme-a form of con- $\mathbf{1 1 2}$ text dependency. Such letter combinations have been 113 called "functional spelling units" (Venezky, 1970; Colt- 114 heart, 1984). Examples of functional spelling units are $\mathbf{1 1 5}$ th $\rightarrow / \mathrm{d} /$ as in that, ch $\rightarrow / \mathrm{t} \mathrm{J} /$ as in church, and $q u \rightarrow / \mathrm{k} / \mathbf{1 1 6}$ as in this example of quay. Unfortunately, any of the let- $\mathbf{1 1 7}$ ters of the functional spelling unit could plausibly align $\mathbf{1 1 8}$ with the corresponding phoneme, with the others corre- 119 sponding to nulls, leading to a degree of indeterminacy. $\mathbf{1 2 0}$

More rarely, there are fewer letters than phonemes $\mathbf{1 2 1}$ in a word of English. Examples are (six,/sIks/) and $\mathbf{1 2 2}$ $(\operatorname{sex}, / \mathrm{s} \varepsilon \mathrm{ks} /)$ in which the single letter $x$ maps to the $\mathbf{1 2 3}$ two phonemes $/ \mathrm{ks} /$, so that 'null letters' may have to $\mathbf{1 2 4}$ be introduced to maintain a one-to-one mapping. Se- 125 jnowski and Rosenberg (1987) actually invented 'new' 126 phonemes $(/ \mathrm{K} /, / \mathrm{X} /$ and $/ \# /)$ in NETtalk to avoid intro- $\mathbf{1 2 7}$ ducing null letters. As with null phonemes, the prob- $\mathbf{1 2 8}$ lem arises as to exactly where the nulls should be $\mathbf{1 2 9}$ 
placed. Worse yet, both problems - null letters and null phonemes - can occur in the same word, as in the case of (axe, /aks/) for which a reasonable alignment is:

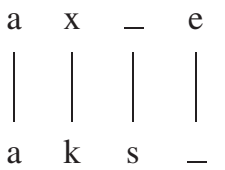

So the simple-minded presumption that the same number of letters and of phonemes implies a one-to-one mapping is mistaken in this case.

These examples illustrate that there is no canonically correct alignment of text and phonemes in every case, nor should we expect this, since the process is essentially a computational convenience lacking any sound linguistic or theoretical basis. The alignment problem is especially severe for languages like English and French whose writing systems are 'deep', i.e., they display a complex relation between spelling and sound lacking consistency and transparency, unlike the 'shallow' orthographies of Finnish or Serbian for example, where the correspondence is mostly if not entirely consistent and transparent (Coltheart, 1978; Liberman et al., 1980; Katz and Feldman, 1981; Turvey et al., 1984; Sampson, 1985). Indeed, (Abercrombie, 1981, p. 209) describes the English spelling-to-sound system as "... one of the least successful applications of the Roman alphabet."

As one last illustration of the complexities of spelling-sound correspondence in English, consider the word (made, $/ \mathrm{meId} /$ ):

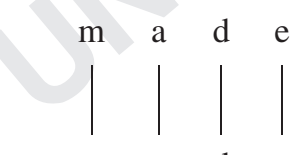

Here, the final $e$ aligns with a null phoneme, yet it does not seem natural to view $d e$ as a functional spelling unit in this case. Removing the $e$ yields the word $(\mathrm{mad}, / \mathrm{mad} /)$, so that it acts as a 'marking' (Venezky, 1970), signifying that the preceding vowel is lengthened or dipthongized: /a/ becomes /eI/. This contrasts with the final $e$ of axe, which has no such marking effect, further illustrating the inconsistent and partly-arbitrary nature of the English spelling system. Markings in English, whereby a final letter affects the sound of a medial vowel letter, can be very long range, as in the well-known example word pairs photograph/photography and telegraph/telegraphy
(Chomsky and Halle, 1968). They can be seen as an $\mathbf{1 7 1}$ interaction of the lack of consistency and transparency, 172 both of which — as we have seen - complicate the pro- $\mathbf{1 7 3}$ cess of alignment.

Given these difficulties, it is clear that the automatic $\mathbf{1 7 5}$ alignment of text and phonemes is not a straightforward $\mathbf{1 7 6}$ matter. In the remainder of this paper, we develop an 177 approach to alignment based on ideas originally found $\mathbf{1 7 8}$ in Luk and Damper (1991, 1992, 1993, 1996), but us- 179 ing much-improved algorithms. Although imperfect, 180 our earlier methods have in fact been used by other au- $\mathbf{1 8 1}$ thors (e.g., Parfitt and Sharman, 1991;Jansche, 2001), 182 reflecting the widespread need for a good alignment $\mathbf{1 8 3}$ algorithm.

\section{Alignment by Dynamic Programming}

Dynamic programming (Bellman, 1957; Kruskal, 186 1983) offers a simple and powerful way to align text $\mathbf{1 8 7}$ and phonemes on the assumption that we have some $\mathbf{1 8 8}$ knowledge of the probability of a particular letter map- 189 ping to a particular phoneme. In this work, knowl- 190 edge about letter-phoneme mappings will be compiled 191 in an 'association' matrix, $\mathbf{A}$, of dimension $L \times P, 192$ where $L$ is the size of the letter inventory (i.e., 26) 193 and $P$ is the size of the phoneme inventory (which 194 is 44 here). The dynamic programming (DP) princi- 195 ple asserts that the global solution to a path-finding $\mathbf{1 9 6}$ problem can be found by a sequence of locally-optimal 197 steps; in other words, no local non-optimality can con- 198 tribute to a globally-optimal solution. This principle is $\mathbf{1 9 9}$ well-known and widely-used in computational linguis- 200 tics and speech technology, forming for instance the 201 basis of the CYK parsing algorithm (Hopcroft et al., 202 2001, pp. 298-301) and the Viterbi algorithm (Viterbi, 203 1967; Forney, 1973; Neuhoff, 1975), used in various 204 guises in speech recognition, speech synthesis, and text $\mathbf{2 0 5}$ processing. $\quad 206$

The process of aligning text and phonemes for a spe- 207 cific word can be cast as a path-finding problem by $\mathbf{2 0 8}$ building a table, or B matrix, indexed by the letters of 209 the word's spelling and the phonemes of its pronun- $\mathbf{2 1 0}$ ciation. This is illustrated for the word (phase,/feIz/) 211 in Fig. 1(a). The entries in this matrix are to be inter- 212 preted as degrees of 'association' between each letter 213 and each phoneme. The procedure for inferring these $\mathbf{2 1 4}$ entries is detailed in later sections. (The values seen $\mathbf{2 1 5}$ here are taken from one iteration of an actual run of our $\mathbf{2 1 6}$ algorithm.) Note that we have added word delimiters 217 (\# and \$ for letter and phoneme domains respectively), $2 \mathbf{2 1 8}$ 
with an association of 0 for $(\#, \$)$. This is done to allow the DP algorithm to align the leading letter or phoneme of a word with a null; otherwise the first letter would always align with the first phoneme. The 'best' alignment of letters and phonemes is then defined by the path from the top-left entry of the matrix to the bottom-right that maximizes the accumulation of association values along this path.

To find this best alignment, we introduce two new matrices $\mathbf{C}$ and $\mathbf{D}$. Matrix $\mathbf{C}$ is a table of accumulated associations, such that each entry is the maximum accumulated association up to that point in the table (i.e., up to that point in the alignment). Matrix $\mathbf{D}$ holds pointers indicating the precursor cell from which the DP algorithm moved to each cell. The $\mathbf{C}$ and $\mathbf{D}$ matrices are filled left-to-right, top-to-bottom using some appropriate form of simple recursive maximization equation. At the end of the process, the $\mathbf{C}$ matrix holds the maximum accumulated association for the complete word in its bottom right cell, and the best alignment can be found by tracing pointers back from the bottom right cell of the $\mathbf{D}$ matrix.

In this work, we have used the implementation of DP due to Needleman and Wunsch (1970), since it is simple, well-known and performed very satisfactorily in preliminary, exploratory investigations. The specific form of the recursive maximization equation for a given word $w$ is:

$$
C_{i, j}=\max \left\{\begin{array}{l}
C_{i-1, j-1}+B_{i, j}, \\
C_{i-1, j}-\delta, \\
C_{i, j-1}-\delta
\end{array}\right\} \quad \begin{aligned}
& 1 \leq i \leq\left|l_{w}\right| \\
& 1 \leq j \leq\left|p_{w}\right|
\end{aligned}
$$

where $\left|l_{w}\right|$ and $\left|p_{w}\right|$ are the lengths of word $w$ in terms of letters and phonemes (including delimiters) respectively, and $\delta$ is some suitably chosen penalty term, which here is set to 0 .

Figure 1(b) shows the $\mathbf{C}$ and $\mathbf{D}$ matrices found for the word (phase,/feIz/) with the associations tabulated in Fig. 1(a). For ease of illustration, the two matrices are shown superimposed. If the maximization chose the $C_{i-1, j-1}+B_{i, j}$ argument, corresponding to a diagonal move in the $\mathbf{B}$ and $\mathbf{C}$ matrices, the entry in the D matrix is "У". If the maximization chose the $C_{i-1, j}$ argument, corresponding to a vertical move in the $\mathbf{B}$ and $\mathbf{C}$ matrices, the entry in the D matrix is " $\downarrow$ ", corresponding to alignment of a letter with a null phoneme. If the maximization chose the $C_{i, j-1}$ argument, corresponding to a horizontal move in the $\mathbf{B}$ and $\mathbf{C}$ matrices, the entry in the $\mathbf{D}$ matrix is " $\rightarrow$ ", corresponding to alignment of a phoneme with a null

letter. The " $\epsilon$ " in the top left cell indicates the start 259 for the DP alignment from which no back-tracing is $\mathbf{2 6 0}$ possible. The maximal association (or DP score) for $\mathbf{2 6 1}$ the word is align $($ phase $)=71446$. By tracing point- $\mathbf{2 6 2}$ ers back from the bottom right entry, the alignment $\mathbf{2 6 3}$ is found as:

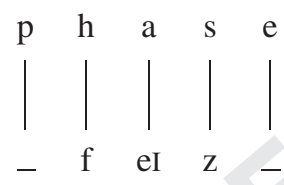

Note that the dynamic programming handles con- 266 text dependency (e.g., letter group $p h$ acts here as $\mathbf{2 6 7}$ a functional spelling unit) in an implicit manner, 268 since at each step of the maximization, Eq. (1), 269 we consider moves from the three possible pre- $\mathbf{2 7 0}$ cursors (cells $(i-1, j-1),(i-1, j)$, and $(i, j-1)) \quad 271$ of cell $(i, j)$. At the same time, the very strong $\mathbf{2 7 2}$ $a \rightarrow / \mathrm{eI} /$ and $s \rightarrow$ /z/ associations of 23098 and $45788 \mathbf{2 7 3}$ respectively in Fig. 1 act as 'anchors' for the DP 274 alignment.

It only remains to find the A matrix and thereafter $\mathbf{2 7 6}$ we can align any word in the dictionary. This is done $\mathbf{2 7 7}$

\begin{tabular}{|c||c|r|r|r|r|}
\hline & $\$$ & \multicolumn{1}{c|}{$\mathrm{f}$} & \multicolumn{1}{c|}{ eI } & \multicolumn{1}{c|}{$\mathrm{z}$} & $\$$ \\
\hline \hline$\#$ & 0 & 0 & 0 & 0 & 0 \\
\hline $\mathrm{p}$ & 0 & 9 & 0 & 0 & 0 \\
\hline $\mathrm{h}$ & 0 & 2580 & 27 & 35 & 0 \\
\hline $\mathrm{a}$ & 0 & 42 & 23098 & 937 & 0 \\
\hline $\mathrm{s}$ & 0 & 79 & 3 & 45788 & 0 \\
\hline $\mathrm{e}$ & 0 & 947 & 1732 & 2641 & 0 \\
\hline$\#$ & 0 & 0 & 0 & 0 & 0 \\
\hline
\end{tabular}

(a)

\begin{tabular}{|c||c|r|r|r|r|}
\hline & S & \multicolumn{1}{c|}{ f } & \multicolumn{1}{c|}{ eI } & \multicolumn{1}{c|}{$\mathrm{z}$} & \multicolumn{1}{c|}{ S } \\
\hline \hline$\#$ & $0, \epsilon$ & $0, \rightarrow$ & $0, \rightarrow$ & $0, \rightarrow$ & $0, \rightarrow$ \\
\hline $\mathrm{p}$ & $0, \downarrow$ & $9, \searrow$ & $9, \rightarrow$ & $9, \rightarrow$ & $9, \rightarrow$ \\
\hline $\mathrm{h}$ & $0, \downarrow$ & $2580, \searrow$ & $2580, \rightarrow$ & $2580, \rightarrow$ & $2580, \rightarrow$ \\
\hline $\mathrm{a}$ & $0, \downarrow$ & $2580, \downarrow$ & $25678, \searrow$ & $25678, \rightarrow$ & $25678, \rightarrow$ \\
\hline $\mathrm{s}$ & $0, \downarrow$ & $2580, \downarrow$ & $25678, \downarrow$ & $71446, \searrow$ & $71446, \rightarrow$ \\
\hline $\mathrm{e}$ & $0, \downarrow$ & $2580, \downarrow$ & $25678, \downarrow$ & $71446, \downarrow$ & $71446, \searrow$ \\
\hline$\#$ & $0, \downarrow$ & $2580, \downarrow$ & $25678, \downarrow$ & $71446, \downarrow$ & $71446, \searrow$ \\
\hline
\end{tabular}

(b)

Figure 1. (a) Example matrix of letter-phoneme associations (B matrix) for the word (phase,/feIz/). The word is delimited by \# and $\$$ in the letter and phoneme domains respectively. See text for explanation of entries. (b) Table of cumulative associations found by dynamic programming, together with the production or 'move' from the precursor cell that maximizes this value. This table can be viewed as a superposition of $\mathbf{C}$ and $\mathbf{D}$ matrices (see text). 
using a form of the EM algorithm, which is the subject of the next section.

\section{Estimating Associations with the EM Algorithm}

The expectation-maximum (EM) algorithm is an iterative approach to the solution of maximum-likelihood estimation problems when there are data missing from the set of observations and/or the likelihood function cannot be easily differentiated to find its maxima. Although the basic idea had appeared in the literature previously (e.g., Hartley, 1958; Baum, 1972), the term "EM algorithm" was coined by Dempster et al. (1977). A useful introduction is provided by Moon (1996); an excellent survey and treatment of recent developments is given by McLachlan and Krishnan (1997).

The EM algorithm interleaves two steps, starting from initial, assumed values for the missing data:

1. the $E$-step, in which the expected value of the likelihood is found with respect to the unknown values, using the current estimate of the parameters, conditioned on the observations.

2. the $M$-step, in which this expectation is maximized to yield a new set of parameters.

The E- and M-steps are iterated with each iteration guaranteed to increase the likelihood until we converge to a local maximum of the likelihood function. Convergence is proved by Dempster et al. (1977) and Wu (1983) among others. Like other optimisation techniques that find local maxima by gradient ascent, the particular local maximum found in general depends on the start point of the iteration-i.e., the assumed initial values of the missing data.

In the specific case of letter-phoneme alignment, the observed data are the words listed in the dictionary in terms of their paired spellings/pronunciations. The missing data are the parameters describing the probabilistic correspondence between words and letters that underlie the alignment process and that are compiled into matrix A. As mentioned in Section 4 below, we maximize not the likelihood for word $w$ at iteration $k$ but the maximal DP score (as described in the previous section) given the association matrix from the iteration. Hence, the process must start with an association matrix $\mathbf{A}^{0}$ initialized with some appropriate values.

The simplest way to obtain $\mathbf{A}^{0}$ is the naïve initialization, found as follows. Processing each word of the dictionary in turn, every time a letter $l$ and a phoneme $p \quad 324$ appear in the same word, irrespective of relative po- $\mathbf{3 2 5}$ sition, the corresponding element $a_{l p}^{0}$ of $\mathbf{A}^{0}$ is incre- 326 mented. After the first pass through the dictionary, each $\mathbf{3 2 7}$ element $a_{l p}^{0}$ contains a count of the number of times let- $\mathbf{3 2 8}$ ter $l$ and phoneme $p$ appear in the same word. This is $\mathbf{3 2 9}$ not of course to say that a specific $l$ and $p$ do align; the $\mathbf{3 3 0}$ rationale is that they can only align if they occur in the $\mathbf{3 3 1}$ same word. Although we do not expect this to give a $\mathbf{3 3 2}$ very good estimate of $\mathbf{A}$, an initial alignment can be $\mathbf{3 3 3}$ attempted from $\mathbf{A}^{0}$.

Once we have this (imperfect) alignment, we can per- 335 form a second pass through the dictionary to produce a $\mathbf{3 3 6}$ new and better association matrix $\mathbf{A}^{1}$ with elements $a_{l p}^{1} \quad \mathbf{3 3 7}$ that count the number of times letter $l$ and phoneme $p \mathbf{3 3 8}$ appear at the same (aligned) position, $i$. At this first 339 iteration, nulls are now introduced into the dictionary $\mathbf{3 4 0}$ as a consequence of the DP matching so that letters $\mathbf{3 4 1}$ can associate with null phonemes and phonemes can $\mathbf{3 4 2}$ associate with null letters. Although these nulls obvi- $\mathbf{3 4 3}$ ously affect the counts of letter-phoneme associations, 344 they are not themselves entered as part of the updated $\mathbf{3 4 5}$ matrix $\mathbf{A}^{1}$. They are omitted because to do so worked 346 far better than including nulls. If we include nulls in $\mathbf{3 4 7}$ the set of letters and phonemes at the EM stage, we are $\mathbf{3 4 8}$ effectively building in an unnatural tendency for align- 349 ments to exploit nulls, because of their cumulative high $\mathbf{3 5 0}$ scoring over a variety of situations. Hence, we restrict 351 the role of the nulls to the DP matching stage. 352

Proceeding as above, a new set of candidate align- $\mathbf{3 5 3}$ ments can now be produced and scored, a new 'best' 354 alignment again selected, and $\mathbf{A}^{1}$ updated to $\mathbf{A}^{2}$. Fur- 355 ther iterations can then be used to improve the align- $\mathbf{3 5 6}$ ments, and the estimates of the association matrix, 357 until convergence. $\quad 358$

By its use of a step in which expectations of new cor- 359 respondences are computed (using the current estimate $\mathbf{3 6 0}$ of the correspondences conditioned on the dictionary $\mathbf{3 6 1}$ data) followed by a maximization step, this can be seen $\mathbf{3 6 2}$ as an EM-like algorithm.

\section{Issues with the Alignment Algorithm}

Many interesting issues arise with respect to alignment $\mathbf{3 6 5}$ based on the EM and DP algorithms. In this section, 366 we briefly discuss the more important of them. $\quad 367$

As a form of gradient ascent procedure, convergence $\mathbf{3 6 8}$ is to a local maximum that in general depends upon the $\mathbf{3 6 9}$ start point, i.e., the matrix $\mathbf{A}^{0}$. One possible start point $\mathbf{3 7 0}$ uses the simple naïve approach of the previous section. 371 
Intuitively, this has the disadvantage of allowing any letter to associate with any phoneme, no matter that one might appear at the beginning of a long word and the other at the end. Hence, an attractive possibility is to weight the entries $a_{l p}^{0}$ inversely according to the difference of the position indices of the $l$ and $p$ symbols. For example, the position-index difference between letter $h$ and phoneme /z/ of (phase, /feIz/) is $|2-3|=1$. Various weighting schemes could be envisaged. Yet another possibility is to use the manual alignments devised for training NETtalk (Sejnowski and Rosenberg, 1987) or NETspeak (McCulloch et al., 1987) to obtain $\mathbf{A}^{0}$. (In this latter case, the counts entered into $\mathbf{A}^{0}$ will have taken account of nulls.) Further, Black et al. (1998) have described a similar algorithm to ours in which they specify a set of "allowables", i.e., letters and phonemes that can plausibly associate on the basis of prior intuitive knowledge of letter-phoneme correspondences. This can be used to define binary values for $a_{l p}^{0}$ (which become continuous on subsequent EM iterations). One of the major aims of this paper was to evaluate the wide variety of possibilities for initialization (see Section 5.2).

One very important issue is evaluating quantitatively the effectiveness of any alignment algorithm. However, this is difficult since there is no canonically correct 'gold standard' alignment in all cases (see Introduction). Scoring on the basis of human judgement is likely to be subjective and inconsistent between judges and is, in any case, not practical for the sort of very large dictionaries that we wish to use. Although it is possible (and indeed sensible) to have a human expert check obvious problem cases (e.g., axe, know, phase,...), and we did in fact do this during program development, it does not amount to a full and thorough evaluation, giving a global summary figure of merit. Thus, we have decided to assess our alignment results indirectly according to the number of words correctly transcribed by a pronunciation by analogy $(\mathrm{PbA})$ system. For this purpose, we have used the PbA system of 2000.

Another issue is what we have previously called the 'harmonization' of the different phoneme inventories used by different researchers and/or dictionary compilers (Damper et al., 1999). Thus, if we wish to use the NETtalk manual alignment to estimate $\mathbf{A}^{0}$ in order to align a dictionary such as BEEP (see below), we must have some way of mapping the different sets of phonemes used by the different dictionaries onto a common set. Because our goal is to align BEEP, we obviously choose the BEEP symbols as the common set. Tables 1 and 2 show the harmonization scheme
Table 1. Harmonization scheme used to map the NETtalk phoneme set onto the BEEP set.

\begin{tabular}{|c|c|c|c|}
\hline NETtalk & BEEP & as in $\ldots$ & IPA \\
\hline $\mathrm{a}$ & aа & father & $\mathrm{a}$ \\
\hline $\mathrm{b}$ & $\mathrm{b}$ & bet & $\mathrm{b}$ \\
\hline $\mathrm{c}$ & ao & bought & 0 \\
\hline $\mathrm{d}$ & d & dime & d \\
\hline $\mathrm{e}$ & ey & bake & eI \\
\hline $\mathrm{f}$ & $\mathrm{f}$ & fin & $\mathrm{f}$ \\
\hline $\mathrm{g}$ & $\mathrm{g}$ & guess & g \\
\hline h & hh & head & $\mathrm{h}$ \\
\hline $\mathrm{i}$ & iy & peat & i \\
\hline $\mathrm{k}$ & $\mathrm{k}$ & kitten & $\mathrm{k}$ \\
\hline 1 & 1 & let & 1 \\
\hline $\mathrm{m}$ & $\mathrm{m}$ & met & $\mathrm{m}$ \\
\hline $\mathrm{n}$ & $\mathrm{n}$ & net & $\mathrm{n}$ \\
\hline o & ow & boat & $\mathrm{oU}$ \\
\hline $\mathrm{p}$ & $\mathrm{p}$ & pet & $\mathrm{p}$ \\
\hline $\mathrm{r}$ & $\mathrm{r}$ & red & $\mathrm{r}$ \\
\hline $\mathrm{s}$ & $\mathrm{s}$ & set & $\mathrm{s}$ \\
\hline $\mathrm{t}$ & $\mathrm{t}$ & test & $\mathrm{t}$ \\
\hline $\mathrm{u}$ & uw & lute & $\mathrm{u}$ \\
\hline $\mathrm{v}$ & $\mathrm{v}$ & vest & $\mathrm{v}$ \\
\hline w & w & wet & w \\
\hline $\mathrm{x}$ & $a x$ & about & $\partial$ \\
\hline y & $\mathrm{y}$ & yet & $\mathrm{j}$ \\
\hline $\mathrm{z}$ & $\mathrm{z}$ & zoo & $\mathrm{z}$ \\
\hline A & ay & bite & aI \\
\hline $\mathrm{C}$ & ch & $\underline{\operatorname{chin}}$ & $\mathrm{t} \int$ \\
\hline $\mathrm{D}$ & $\mathrm{dh}$ & this & ð \\
\hline E & eh & bet & $\varepsilon$ \\
\hline G & ng & sing & $\eta$ \\
\hline I & ih & bit & I \\
\hline $\mathrm{J}$ & jh & gin & $d s$ \\
\hline K & $\mathrm{k} \mathrm{s}$ & sexual & $\mathrm{k} \int$ \\
\hline $\mathrm{L}$ & 1 & bottle & $t$ \\
\hline M & $\mathrm{m}$ & abysm & (ə)m \\
\hline $\mathrm{N}$ & $\mathrm{n}$ & button & (ə)n $n$ \\
\hline $\mathrm{O}$ & oy & boy & DI \\
\hline Q & $\mathrm{kw}$ & quest & $\mathrm{k} \mathrm{w}$ \\
\hline $\mathrm{R}$ & er & bird & 3 \\
\hline S & sh & $\underline{\operatorname{shin}}$ & $\int$ \\
\hline $\mathrm{T}$ & th & thin & $\theta$ \\
\hline $\mathrm{U}$ & uh & book & $U$ \\
\hline W & aw & bout & $\mathrm{aU}$ \\
\hline
\end{tabular}


Table 1. (Continue).

\begin{tabular}{|c|c|c|c|}
\hline NETtalk & BEEP & as in ... & IPA \\
\hline $\mathrm{X}$ & $\mathrm{k} \mathrm{s}$ & $\operatorname{sex}$ & $\mathrm{k} \mathrm{s}$ \\
\hline $\mathrm{Y}$ & y uw & cute & $\mathrm{ju}$ \\
\hline $\mathrm{Z}$ & $\mathrm{zh}$ & leisure & 3 \\
\hline @ & ae & bat & $\mathrm{a}$ \\
\hline$!$ & $\mathrm{ts}$ & nazi & $\mathrm{ts}$ \\
\hline \# & $\mathrm{g} \mathrm{z}$ & examine & $\mathrm{gz}$ \\
\hline+ & w aa & bourgeois & w a \\
\hline * & $\mathrm{w}$ & whack & $M$ \\
\hline$\hat{\imath}$ & ah & but & $\Lambda$ \\
\hline
\end{tabular}

used to map the NETtalk and NETspeak phoneme sets onto BEEP. Note that BEEP uses a phoneme inventory of 44 symbols (excluding the null phoneme), whereas the NETtalk and NETspeak inventories are both of size 51 (again excluding the null phoneme).

The symbols listed in the 'NETtalk' column of Table 1 are those in the file downloaded from http: / / www. speech.Cs. cmu . edu/comp. speech and not the ones tabulated in Appendix A of 1987. The

downloaded file includes a symbol ' + ' which is not

433 listed in the paper. In general, harmonization can never

434 be an exact process, because of idiosyncratic choice of

435 phoneme inventories by the different individual com-

436 pilers of the transcribed dictionaries, which often re-

437 flect dialectal differences. For instance, Sejnowski and

438 Rosenberg (1987) use the same symbol /a/ to transcribe

439 both the a vowel in father and the $\mathrm{D}$ vowel in stock, as

440 these are probably the same vowel for their dialect of

441 American English. So the mapping from NETtalk to

442 BEEP symbols is not one-to-one. We can only try to

443 achieve the most consistent mapping according to our 444 intuitions.

445 A final issue is that the EM algorithm is properly a 446 probabilistic algorithm. We experimented with various

447 normalizations, corresponding to various probabilistic

448 models, but none performed as well as using simple

449 (unnormalized) frequency counts directly from the as-

450 sociation matrix A. Hence, all results presented here

451 use this formulation. This is the reason we refer to our

452 algorithm as "EM-like". The effect of using unnormal-

453 ized counts (rather than proper probabilities) on con-

454 vergence is unknown but, as we shall see, this did not 455 prove to be an issue in practice.
Table 2. Harmonization scheme used to map the NETspeak phoneme set onto the BEEP set.

\begin{tabular}{|c|c|c|c|}
\hline NETspeak & BEEP & as in $\ldots$ & IPA \\
\hline A & $\mathrm{ax}$ & about & $\partial$ \\
\hline B & $\mathrm{b}$ & bet & $\mathrm{b}$ \\
\hline D & $\mathrm{d}$ & dime & d \\
\hline E & eh & bet & $\varepsilon$ \\
\hline $\mathrm{F}$ & $\mathrm{f}$ & $\underline{\text { fin }}$ & $\mathrm{f}$ \\
\hline G & $\mathrm{g}$ & guess & $g$ \\
\hline $\mathrm{H}$ & hh & head & $\mathrm{h}$ \\
\hline I & ih & bit & I \\
\hline $\mathrm{J}$ & jh & gin & $d s$ \\
\hline K & $\mathrm{k}$ & kitten & $\mathrm{k}$ \\
\hline $\mathrm{L}$ & 1 & let & 1 \\
\hline M & $\mathrm{m}$ & $\underline{\text { met }}$ & $\mathrm{m}$ \\
\hline $\mathrm{N}$ & $\mathrm{n}$ & net & $\mathrm{n}$ \\
\hline $\mathrm{O}$ & oh & stock & $\mathrm{D}$ \\
\hline $\mathrm{P}$ & $\mathrm{p}$ & $\underline{\text { pet }}$ & $\mathrm{p}$ \\
\hline $\mathrm{R}$ & $\mathrm{r}$ & red & $\mathrm{r}$ \\
\hline S & $\mathrm{s}$ & set & $\mathrm{s}$ \\
\hline $\mathrm{T}$ & $\mathrm{t}$ & test & $\mathrm{t}$ \\
\hline $\mathrm{U}$ & ah & but & $\Lambda$ \\
\hline $\mathrm{V}$ & $\mathrm{v}$ & vest & $\mathrm{v}$ \\
\hline W & w & wet & w \\
\hline Y & $\mathrm{y}$ & yet & $\mathrm{j}$ \\
\hline $\mathrm{Z}$ & $\mathrm{z}$ & zoo & $\mathrm{z}$ \\
\hline AA & ae & bat & $\mathrm{a}$ \\
\hline AI & ey & bake & eI \\
\hline AR & aa & father & $\mathrm{a}$ \\
\hline AW & ao & bought & 0 \\
\hline $\mathrm{CH}$ & $\mathrm{ch}$ & $\underline{\text { chin }}$ & $t \int$ \\
\hline DH & dh & this & ð \\
\hline $\mathrm{EE}$ & iy & peat & $\mathrm{i}$ \\
\hline EI & ea & $\underline{\text { air }}$ & $\varepsilon \partial$ \\
\hline ER & er & bird & 3 \\
\hline EY & ih & despite & I \\
\hline GZ & $\mathrm{g} \mathrm{z}$ & examine & $\mathrm{g} \mathrm{z}$ \\
\hline IA & ia & $\underline{\text { ear }}$ & Іә \\
\hline IE & ay & bite & aI \\
\hline KH & $\mathrm{k} \mathrm{sh}$ & anxious & $\mathrm{k} \int$ \\
\hline KS & $\mathrm{k} \mathrm{s}$ & $\operatorname{sex}$ & $\mathrm{k} \mathrm{s}$ \\
\hline $\mathrm{KW}$ & $\mathrm{kw}$ & quest & $\mathrm{kw}$ \\
\hline NG & ng & sing & $\eta$ \\
\hline OA & ow & boat & oU \\
\hline OI & oy & boy & DI \\
\hline
\end{tabular}


Table 2. (Cоntinue).

\begin{tabular}{lccc}
\hline NETspeak & BEEP & as in ... & IPA \\
\hline OO & uh & book & U \\
OU & aw & bout & aU \\
SH & sh & $\underline{\text { shin }}$ & $\int$ \\
TH & th & $\underline{\text { thin }}$ & $\theta$ \\
UL & 1 & bottle & $t$ \\
UR & ua & moor & luzte \\
UU & uw & cute & u \\
YU & y uw & leisure & 3 \\
ZH & zh & & 3 \\
\hline
\end{tabular}

\section{Results}

457 In this section, we report the results of using our algorithm to align a large dictionary.

\subsection{Initializations}

481 The following initializations were used:

482 - naïve;

483 a weighted scheme with $W=\beta /(1+|d|)$ where $d$ is the letter-phoneme position-index difference, and $\beta$ is a heuristic scaling set to 40 for the results $\mathbf{4 8 4}$ reported here; $\quad \mathbf{4 8 5}$

- the NETtalk manual alignment (20,009 words); $4 \mathbf{4 8 6}$

- the NETspeak manual alignment (16,280 words); 487

- various random alignments.

488

\subsection{Convergence}

The convergence criterion was that there was no change 490 as between $\mathbf{A}^{k}$ and $\mathbf{A}^{k-1}$.

Figure 2 shows the convergence behavior for the $\mathbf{4 9 2}$ NETtalk initialisation. The quantity graphed is the to- 493 tal DP score for the whole dictionary at the end of 494 iteration $k$, i.e., $S_{k}=\sum_{i=1}^{198,632} \operatorname{align}^{k}\left(w_{i}\right)$. Note that 495 convergence requires that the A matrix is unchanged 496 between iterations, $\mathbf{A}^{k}=\mathbf{A}^{k-1}$, which (because nulls 497 are not included in the $\mathbf{A}$ matrix) is not quite the $\mathbf{4 9 8}$ same as the total DP score remaining unchanged, 499 $S_{k}=S_{k-1}$. The total DP score at the zeroth iteration, $S_{0}, \mathbf{5 0 0}$ is very low in this case, because only the 20,009 words $\mathbf{5 0 1}$ of the originally-aligned NETtalk dictionary can be $\mathbf{5 0 2}$ scored. $\quad 503$

Figure 3 shows convergence behavior for two dif- $\mathbf{5 0 4}$ ferent initializations, excluding the total DP score at $\mathbf{5 0 5}$ the zeroth iteration, $S_{0}$. This gives a clearer view $\mathbf{5 0 6}$ of the convergence for the NETtalk initialization than $\mathbf{5 0 7}$ does Fig. 2 where the very low value of $S_{0}$ swamps $\mathbf{5 0 8}$ the trend. For the naïve initialization, it is not re- $\mathbf{5 0 9}$ ally sensible to depict $S_{0}$ anyway since the dramatic $\mathbf{5 1 0}$ overcounting of associations (every letter is counted $\mathbf{5 1 1}$ $\left|p_{w}\right|$ times and every phoneme is counted $\left|l_{w}\right|$ times) $\mathbf{5 1 2}$ produces a very high score that is effectively mean- $\mathbf{5 1 3}$ ingless. For both initializations, most of the improve- $\mathbf{5 1 4}$ ment takes place between the first and second iter- $\mathbf{5 1 5}$ ations. This was found to be a general characteris- $\mathbf{5 1 6}$ tic of the results. For all initializations, convergence $\mathbf{5 1 7}$ was achieved in between 5 to 8 iterations. The man- $\mathbf{5 1 8}$ ual alignment of the NETtalk dictionary, even though 519 it is much smaller than BEEP, shows a clear ben- $\mathbf{5 2 0}$ efit in terms of a higher score at iteration 1 to- $\mathbf{5 2 1}$ gether with faster convergence. The score at con- $\mathbf{5 2 2}$ vergence, $S_{C}$, was remarkably consistent across the $\mathbf{5 2 3}$ various initializations, suggesting that the search prob- $\mathbf{5 2 4}$ lem is strongly convex. The best value obtained $\mathbf{5 2 5}$ was $S_{C}=8.579 \times 10^{10}$ for the NETtalk initialization $\mathbf{5 2 6}$ whereas the worst value was $S_{C}=8.473 \times 10^{10}$ for $\mathbf{5 2 7}$ one of the random initializations. Generally, the ran- $\mathbf{5 2 8}$ dom initialization values were slightly lower than the $\mathbf{5 2 9}$ others. 


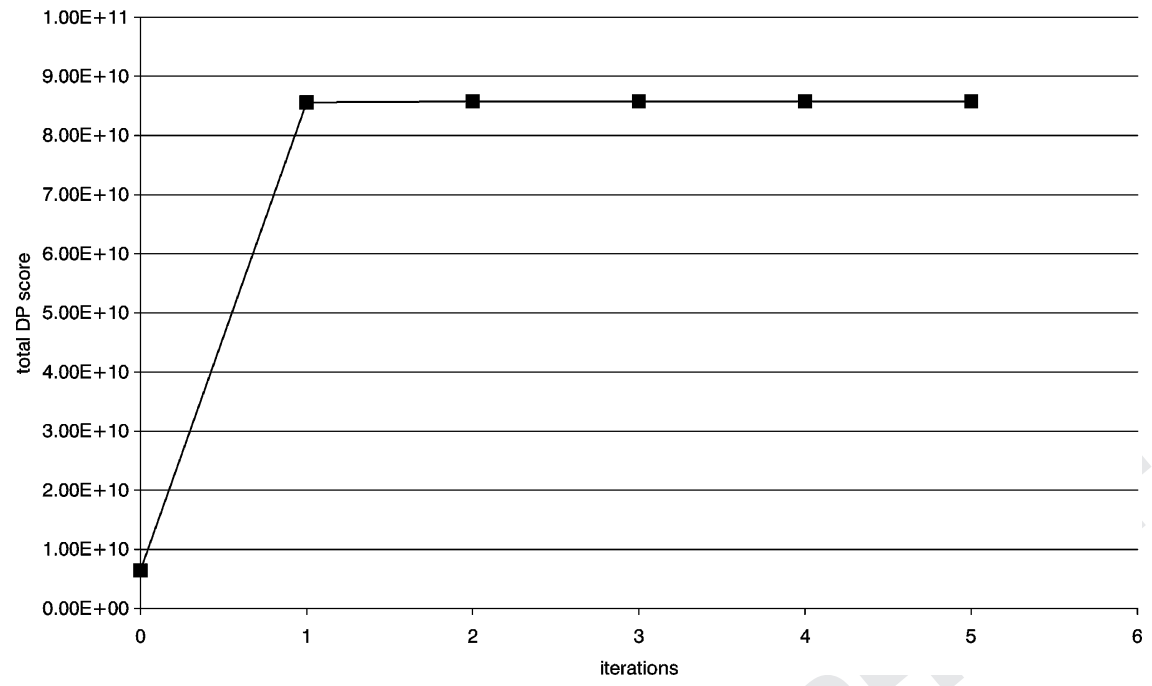

Figure 2. Convergence behavior of the alignment algorithm for the NETtalk initialization.

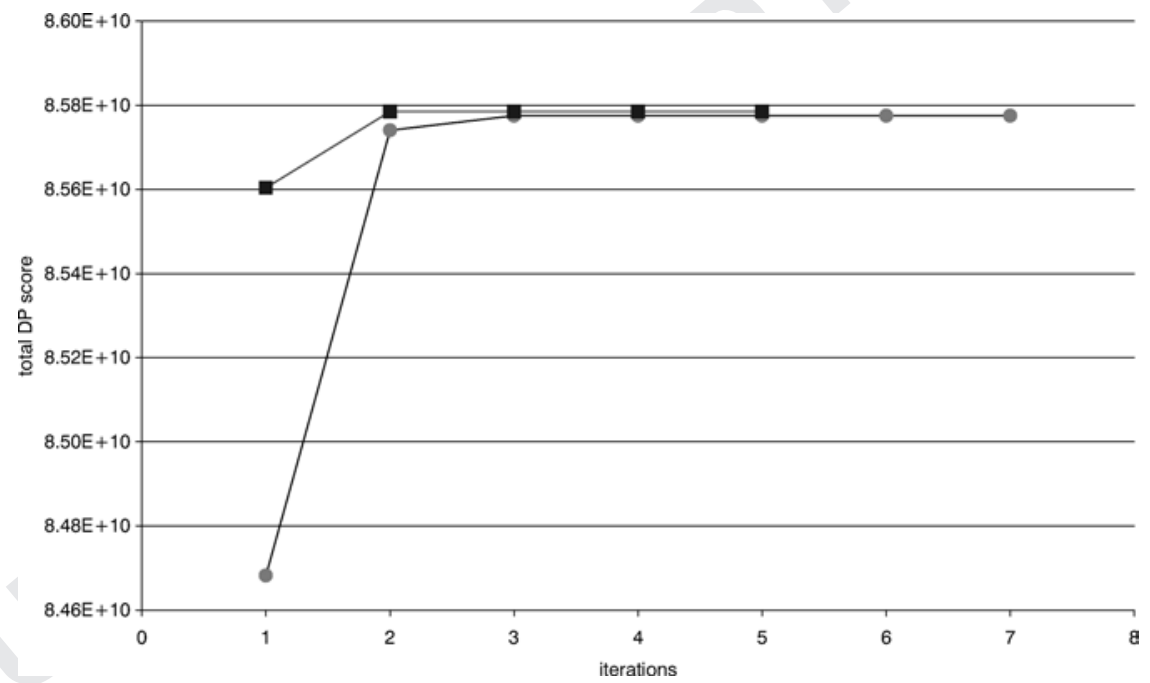

Figure 3. Convergence behavior of the alignment algorithm for two different initializations. Rectangles: NETtalk initialization; Circles: naïve initialization.

\section{5.4. Analysis of Association Matrices}

532 Figures 4(a) and (b) show the association matrices 533 for the naïve initialization initially, $\mathbf{A}^{0}$, and at conver534 gence, $\mathbf{A}^{7}$. The larger association values in Fig. 4 are a consequence of the overcounting mentioned above. As expected, the matrix is considerably less random (i.e., peakier) at convergence. Quantitatively, the (negative) entropy of the $\mathbf{A}^{0}$ matrix was 8.84 bits whereas that of the converged matrix was 5.24 bits; these figures compare with 10.13 bits for the equiprobable case. En- couragingly, the strongest peaks at convergence, corre- $\mathbf{5 4 1}$ sponding to the major letter-phoneme associations, are $\mathbf{5 4 2}$ also among the strongest peaks in $\mathbf{A}^{0}$, indicating that $\mathbf{5 4 3}$ the naïve initialization, albeit very simple, still provides $\mathbf{5 4 4}$ an effective start point for our algorithm.

There is a wealth of information about letter- 546 phoneme correspondences in English to be gleaned $\mathbf{5 4 7}$ from the A matrix obtained at convergence. Since nulls $\mathbf{5 4 8}$ are introduced into the aligned dictionary only at the $\mathbf{5 4 9}$ DP matching stage (see Section 3) and do not figure $\mathbf{5 5 0}$ in the A matrix, they are not considered explicitly in $\mathbf{5 5 1}$ 


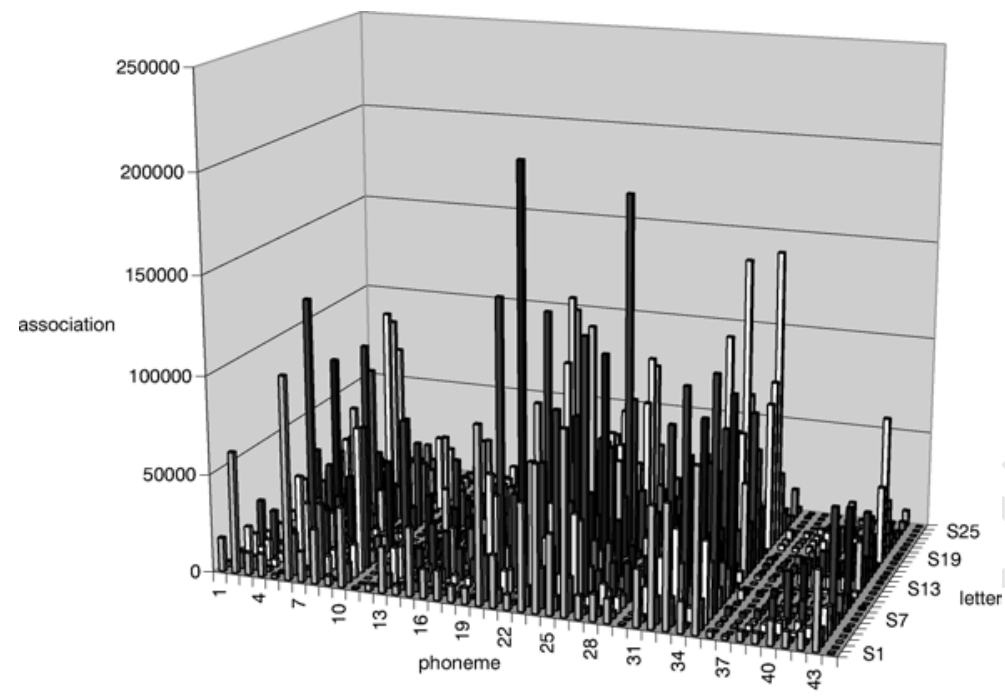

(a)

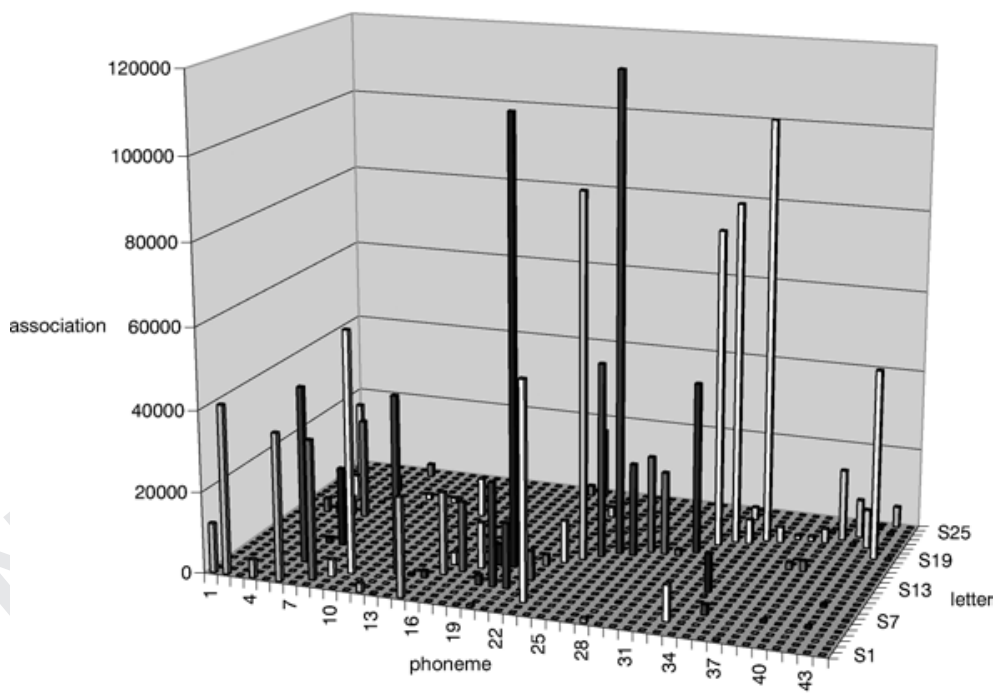

(b)

Figure 4. Association matrices for the naïve initialization both initially, $\mathbf{A}^{0}$, and at convergence, $\mathbf{A}^{7}$.

the remarks that follow. With this proviso, the commonest correspondence overall was $n \rightarrow / \mathrm{n} /$. The commonest letter participating in correspondences is $i$, which occurs 148,913 times in the matrix. This is slightly surprising as the commonest letter overall is $e$. The apparent discrepancy is explained by the number of times letter $e$ participates in a functional spelling unit such as $e a$ and so aligns with null (with the letter $a$ aligning with the vowel phoneme). The least common letter participating in correspondences is $q$, which occurs just 17 times. Again, $q$ almost invariably occurs in a $q u$ functional spelling unit, with $q$ aligning with $\mathbf{5 6 3}$ a null phoneme, which reduces its count in the ma- 564 trix. The commonest phoneme is /I/ at 138,176 occur- $\mathbf{5 6 5}$ rences, which can be understood from the frequency $\mathbf{5 6 6}$ with which letter $i$ occurs and the fact that $i \rightarrow /$ I/ 567 is a very common correspondence (at 109,508 occur- $\mathbf{5 6 8}$ rences). Schwa, /ə/, is relatively less common than /I/ $\mathbf{5 6 9}$ at 190,975 occurrences. Intuitively, one might expect $\mathbf{5 7 0}$ schwa to be the commonest vowel, but it is perhaps $\mathbf{5 7 1}$ more likely than /I/ to align with a null letter. Of the $\mathbf{5 7 2}$ letters, $o$ displays most variability in its association with $\mathbf{5 7 3}$ 
phonemes, with no less than nine correspondences with a frequency count of 1000 or more. The least variability is shown by letter $m$, which almost always associates with phoneme $/ \mathrm{m} /$. Schwa displays easily the most variability in its association with letters, participating in five correspondences (with letters $a, e, i, o$ and $u$ ) with a count greater than 1000 . The least variable phoneme was $/ y /$, which associated with letter $n$ in all but just 2 cases.

\subsection{Assessing Alignment Performance Using PbA}

As previously stated, alignment results were assessed using $\mathrm{PbA}$. Each word was removed from the dictionary and a pronunciation determined from the word's spelling by analogy with all other words. The Marchand and Damper PbA system uses multiple (actually five) criteria to select between candidate pronunciations to find the 'best'. There is, however, a problem in that $\mathrm{PbA}$ was designed to transcribe text in which there will obviously be no null letters. Yet here, null letters have been added to the alignments of many words. Our first step, then, has been to ignore any words with null letters, reducing the number of words to be tested from 198,632 to approximately 177,000 . (The number varies with the exact initialization used.) This is an obvious simplification of the problem, but should nonetheless yield interesting insights.

Table 3 shows results obtained (for words without null letters) in terms of words and phonemes correctly pronounced for each of the initializations used. Several different random initializations were used, but results were very similar and so figures for one only are tabulated here. In each case, we show the results for the best single scoring criterion of the five, for the best combination, and when all five are combined. Note that 10100 in the column heading indicates that scoring strategies 1 and 3 as described by Marchand and Damper (2000, pp. 207-208) provided the best combination performance for all initializations. Although space precludes a full description of our $\mathrm{PbA}$ methodology, we mention that strategy 1 takes the product of arc frequencies along the shortest path in the pronunciation lattice, whereas strategy 3 counts the number of identical pronunciations having the same shortest path length. Strategy 1 is relatively popular in $\mathrm{PbA}$ (e.g., Damper and Eastmond, 1997) whereas we are not aware that any other researchers have ever used strategy 3, which interestingly turns out to be best performing single strategy overall.
Table 3. Results when alignment of the BEEP dictionary is assessed by the performance of a pronunciation by analogy system, for various initializations. Words with null letters in their alignments have been ignored at this stage.

\begin{tabular}{lccc}
\hline & Best Single & Best Combination & All 5 \\
\hline NAïVE & 00100 & 10100 & 11111 \\
Words (\%) & 85.84 & 87.32 & 85.96 \\
Phonemes (\%) & 97.52 & 97.78 & 97.57 \\
$W$ WEIGHTED & 00100 & 10100 & 11111 \\
Words (\%) & 85.87 & 87.36 & 86.00 \\
Phonemes (\%) & 97.60 & 97.85 & 97.65 \\
NETTALK & 00100 & 10100 & 11111 \\
Words (\%) & 86.00 & 87.41 & 86.05 \\
Phonemes (\%) & 97.59 & 97.83 & 97.63 \\
NETSPEAK & 00100 & 10100 & 11111 \\
Words (\%) & 86.01 & 87.48 & 86.11 \\
Phonemes (\%) & 97.64 & 97.89 & 97.70 \\
RANDOM & 00100 & 10100 & 11111 \\
Words (\%) & 85.87 & 87.38 & 85.69 \\
Phonemes (\%) & 97.51 & 97.78 & 97.57 \\
\hline
\end{tabular}

The figures in Table 3 are remarkably consistent, in- $\mathbf{6 2 2}$ dicating that the particular initialization used does not $\mathbf{6 2 3}$ have a dramatic effect. This is in spite of our attempts $\mathbf{6 2 4}$ to restart the algorithm from a variety of very differ- $\mathbf{6 2 5}$ ent points, suggesting that the search space is strongly $\mathbf{6 2 6}$ convex. It is worth noting, however, that as a con- $\mathbf{6 2 7}$ sequence of the large dictionary size (approximately $\mathbf{6 2 8}$ 177,000 words) the difference between the best Best $\mathbf{6 2 9}$ Combination of $87.48 \%$ (for the NETtalk initialization) $\mathbf{6 3 0}$ and the worst Best Combination of $87.32 \%$ (for the $\mathbf{6 3 1}$ naïve initialization) is in fact marginally significant at $\mathbf{6 3 2}$ the 5\% level (binomial test, $z=2.026, p \sim 0.021$ ). $\quad 633$

The best PbA performance is found for NETspeak $\mathbf{6 3 4}$ but initializing alignment with the NETspeak dictionary $\mathbf{6 3 5}$ actually produced a slightly lower total DP score at con- $\mathbf{6 3 6}$ vergence than initializing with NETtalk. In other words, $6 \mathbf{6 3 7}$ the total DP score at convergence is a good but not per- $\mathbf{6 3 8}$ fect indicator of $\mathrm{PbA}$ performance. Examination of the $\mathbf{6 3 9}$ final alignments revealed that these were strongly sim- $\mathbf{6 4 0}$ ilar; there were typically somewhere between 10 and $\mathbf{6 4 1}$ 100 different alignments only between one initializa- $\mathbf{6 4 2}$ tion and another. Most often, differences were due to $\mathbf{6 4 3}$ the specific placement of nulls in words having many $\mathbf{6 4 4}$ silent letters (e.g., bourgeoisie, heavyweight, mem- 645 oirs). Frequently, these were words of foreign (French) $\mathbf{6 4 6}$ origin. 
This is certainly among the best performance fig-

ures ever reported on English letter-phoneme conversion, in terms of word-level accuracy on a large dictionary. Previously (Damper et al., 1999), we obtained $71.8 \%$ words correct using $\mathrm{PbA}$ on a much smaller dictionary - the 16,280 manually-aligned words used by McCulloch et al. (1987) to train NETspeak. (It should be noted, however, that BEEP uses a smaller phoneme inventory of 44 symbols than the 51 used in the NETspeak dictionary, making for a somewhat easier problem.) A further observation is that using all five strategies does not give best performance, as it did for our earlier work with smaller dictionaries (Marchand and Damper, 2000). In assessing performance, however, we must remember that we have simplified the problem by ignoring words with nulls, which arguably gives a too optimistic view of the present results. However, even under the maximally pessimistic assumption that $\mathrm{PbA}$ were to get all the words with null letters wrong, the $85.8 \%$ words correct for best single strategy, naïve start point, would fall to $76.1 \%$ still a very respectable result on such a sizable dictionary.

To gain further insight into this issue, $\mathrm{PbA}$ was used to produce pronunciations for all 198,632 words including those with null letters in their alignment, treating the latter as a legitimate input symbol (even though it never could be in practice). Results for the best combination averaged $82.3 \%$ words correct, showing that high accuracy is potentially achievable if only 'missing' nulls in the $\mathrm{PbA}$ input could be appropriately introduced.

\section{Discussion and Conclusions}

We have described a form of the EM algorithm, used with dynamic programming to align a dictionary of word spellings and their pronunciations. Such alignment problems commonly occur in speech technology and natural language processing. The issues that arise in solving this important problem have been detailed and discussed. The quality of the obtained alignment has been assessed using pronunciation by analogy to derive pronunciations for all words in the dictionary from their spelling, using the aligned data as a knowledge base. Since the EM algorithm is effectively a gradient ascent procedure prone to finding local maxima, alignment has been performed from a variety of initializations, or start points. Results are judged to be extremely encouraging, and are relatively insensitive to a wide variety of start points. This indicates that the search space is strongly convex and, hence, that local $\mathbf{6 9 6}$ maxima are not a practical problem.

Our work has several similarities with that of Ristad $\mathbf{6 9 8}$ and Yianilos (1998). This is perhaps not surprising as 699 they take the topic of stochastic transduction as their $\mathbf{7 0 0}$ motivation, whereas the ideas reported in this paper $\mathbf{7 0 1}$ had their early expression in our own work, which led $\mathbf{7 0 2}$ to the use of stochastic transduction to solve problems $\mathbf{7 0 3}$ in TTS conversion, including letter-phone alignment $\mathbf{7 0 4}$ (Luk and Damper, 1996, 1998). Ristad and Yianilos 705 also use dynamic programming in conjunction with $\mathbf{7 0 6}$ the EM algorithm to learn edit distances between two $\mathbf{7 0 7}$ strings. Since the string edit operations of insertion and $\mathbf{7 0 8}$ deletion can be interpreted as the introduction of nulls $\mathbf{7 0 9}$ into one string or another-either the word's spelling $\mathbf{7 1 0}$ or its pronunciation-there is clearly a strong relation $\mathbf{7 1 1}$ between the two pieces of work. As Jansche (2001) 712 writes: "The problem of letter-to-sound conversion is $\mathbf{7 1 3}$ very similar to the problem of modeling pronunciation $\mathbf{7 1 4}$ variation". However, although Ristad and Yianilos con- 715 sider the problem of pronunciation modelling in speech $\mathbf{7 1 6}$ technology, they do not consider alignment problems $\mathbf{7 1 7}$ as such.

This work represents the most comprehensive study $\mathbf{7 1 9}$ to date of letter-phoneme alignment, at the same time $\mathbf{7 2 0}$ achieving what is probably the best reported perfor- $\mathbf{7 2 1}$ mance on the difficult task of letter-phoneme conver- $\mathbf{7 2 2}$ sion of unknown words of English. Since the aligned $\mathbf{7 2 3}$ BEEP dictionary is a potentially valuable resource, $\mathbf{7 2 4}$ the version obtained from the NETspeak initialization $\mathbf{7 2 5}$ (which produced best performance on letter-phoneme $\mathbf{7 2 6}$ conversion) is made freely available for research $\mathbf{7 2 7}$ use at http://festvox.org/packed/data/ $\mathbf{7 2 8}$ damper. Since our software has wide applicability, we $\mathbf{7 2 9}$ are also working to provide an on-line facility at which $\mathbf{7 3 0}$ researchers can submit dictionaries for alignment.

\section{References}

Abercrombie, D. (1981). Extending the Roman alphabet: Some or- $\mathbf{7 3 3}$ thographic experiments of the past four centuries. In R.E. Asher $\mathbf{7 3 4}$ and E. Henderson (Eds.), Towards a History of Phonetics. Edin- 735 burgh, UK: Edinburgh University Press, pp. 207-224. 736

Bagshaw, P.C. (1998). Phonemic transcription by analogy in text- $\mathbf{7 3 7}$ to-speech synthesis: Novel word pronunciation and lexicon com- $\mathbf{7 3 8}$ pression. Computer Speech and Language, 12(2):119-142. 739

Baum, L.E. (1972). An inequality and associated maximization tech- $\mathbf{7 4 0}$ nique in statistical estimation for probabilistic functions of Markov 741 processes. In Inequalities III: Proceedings of the Third Symposium $\mathbf{7 4 2}$ on Inequalities, Los Angeles, CA, pp. 1-8.

Bellman, R. (1957). Dynamic Programming. Princeton, NJ: Prince- 744 ton University Press. 
Black, A.W., Lenzo, K., and Pagel, V. (1998). Issues in building general letter-to-sound rules. In Proceedings of 3rd European Speech Communication Association (ESCA)/COCOSDA International Workshop on Speech Synthesis. Jenolan Caves, Australia, pp. 77-80.

Carney, E. (1994). A Survey of English Spelling. London, UK: Routledge.

Chomsky, N. and Halle, M. (1968). The Sound Pattern of English. New York, NY: Harper and Row.

Coltheart, M. (1978). Lexical access in simple reading tasks. In G. Underwood (Ed.), Strategies of Information Processing. New York: Academic Press, pp. 151-216.

Coltheart, M. (1984). Writing systems and reading disorders. In L. Henderson (Ed.), Orthographies and Reading: Perspectives from Cognitive Psychology, Neuropsychology and Linguistics. London, UK: Lawrence Erlbaum Associates, pp. 67-79.

Damper, R.I. (Ed.) (2001). Data-Driven Methods in Speech Synthesis. Dordrecht, The Netherlands: Kluwer Academic Publishers.

Damper, R.I. and Eastmond, J.F.G. (1996). Pronouncing text by analogy. In Proceedings of 16th International Conference on Computational Linguistics. Copenhagen, Denmark, Vol 2, pp. 268273.

Damper, R.I. and Eastmond, J.F.G. (1997). Pronunciation by analogy: Impact of implementational choices on performance. Language and Speech, 40(1):1-23.

Damper, R.I., Marchand, Y., Adamson, M.J., and Gustafson, K. (1999). Evaluating the pronunciation component of text-to-speech systems for English: A performance comparison of different approaches. Computer Speech and Language, 13(2):155-176.

Dedina, M.J. and Nusbaum, H.C. (1991). PRONOUNCE: A program for pronunciation by analogy. Computer Speech and Language, 5(1):55-64.

Dempster, A.P., Laird, N.M., and Rubin, D.B. (1977). Maximumlikelihood from incomplete data via the EM algorithm. Journal of the Royal Statistical Society, Series B, 39(1):1-38.

Federici, S., Pirrelli, V., and Yvon, F. (1995). Advances in analogybased learning: False friends and exceptional items in pronunciation by paradigm-driven analogy. In Proceedings of International Joint Conference on Artificial Intelligence (IJCAI'95) Workshop on New Approaches to Learning for Natural Language Processing, Montreal, Canada, pp. 158-163.

Forney, G. D. (1973). The Viterbi algorithm. In Proceedings of the IEEE, vol. 61, no. 3, pp. 268-278.

Hartley, H. (1958). Maximum likelihood estimation from incomplete data. Biometrics 14, 174-194.

Henderson, L. (1984). Writing systems and reading processes. In L. Henderson (Ed.), Orthographies and Reading: Perspectives from Cognitive Psychology, Neuropsychology and Linguistics. London, UK: Lawrence Erlbaum Associates, pp. 11-24.

Hopcroft, J.E., Motwani, R., and Ullman, J.D. (2001). Introduction to Automata Theory, Languages, and Computation, 2nd ed. Boston, MA: Addison-Wesley.

Jansche, M. (2001). Re-engineering letter to sound rules. In Second Meeting of the North American Chapter of the Association for Computational Linguistics, NAACL 2001, Pittsburg, PA. Paper N01-1015 in on-line archive at http://acl.ldc. upenn. edu/N/N01/.

Katz, L. and Feldman, L.B. (1981). Linguistic coding in word recognition: comparisons between a deep and a shallow orthography. In A.M. Lesgold and C.A. Perfetti (Eds.), Interactive Processes in Reading. Hillsdale, NJ: Lawrence Erlbaum Associates, pp. 85- 806 106

Knill, K. and Young, S. (1997). Hidden Markov models in speech $\mathbf{8 0 8}$ and language processing. See Young and Bloothooft (1997), pp. 809 $27-68$.

810

Kruskal, J.B. (1983). An overview of sequence comparison. In 811 D. Sankoff and J. B. Kruskal (Eds.), Time Warps, String Edits $\mathbf{8 1 2}$ and Macromolecules: The Theory and Practice of Sequence Com- 813 parison. Reading, MA: Addison-Wesley, pp. 11-44. $\mathbf{8 1 4}$

Lawrence, S.G.C. and Kaye, G. (1986). Alignment of phonemes 815 with their corresponding orthography. Computer Speech and Lan- $\mathbf{8 1 6}$ guage, 1(2):153-165. 817

Liberman, I., Liberman, A., Mattingly, I. and Shankweiler, D. 818 (1980). Orthography and the beginning reader. In J. Kavanagh 819 and R. Venezky (Eds.), Orthography, Reading and Dyslexia. Bal- 820 timore, OH: University Park Press, pp. 137-153. 821

Luk, R.W.P. and Damper, R.I. (1991). A novel approach to inferring $\mathbf{8 2 2}$ letter-phoneme correspondences. In Proceedings of IEEE Interna- $\mathbf{8 2 3}$ tional Conference on Acoustics, Speech, and Signal Processing, $\mathbf{8 2 4}$ ICASSP'91. Toronto, Canada, Vol 2, pp. 741-744. 825

Luk, R.W.P. and Damper, R.I. (1992). Inference of letter-phoneme $\mathbf{8 2 6}$ correspondences by delimiting and dynamic time warping tech- $\mathbf{8 2 7}$ niques. In Proceedings of IEEE International Conference on $\mathbf{8 2 8}$ Acoustics, Speech, and Signal Processing, ICASSP'92. San Fran- 829 cisco, CA, Vol 2, pp. II.61-II.64.

Luk, R.W.P. and Damper, R.I. (1993). Inference of letter-phoneme $\mathbf{8 3 1}$ correspondences with pre-defined consonant and vowel patterns. 832 In Proceedings of IEEE International Conference on Acoustics, $\mathbf{8 3 3}$ Speech, and Signal Processing, ICASSP'93. Minneapolis, MN, 834 Vol 2, pp. II.203-II.206.

Luk, R.W.P. and Damper, R.I. (1996). Stochastic phonographic trans- 836 duction for English. Computer Speech and Language, 10(2):133- 837 153.

Luk, R.W.P. and Damper, R.I. (1998). Computational complexity of $\mathbf{8 3 9}$ a fast Viterbi decoding algorithm for stochastic letter-phoneme $\mathbf{8 4 0}$ transduction. IEEE Transactions on Speech and Audio Process- 841 ing, 6(3):217-225.

842

Marchand, Y. and Damper, R.I. (2000). A multistrategy approach 843 to improving pronunciation by analogy. Computational Linguis- $\mathbf{8 4 4}$ tics, 26(2):195-219.

McCulloch, N., Bedworth, M., and Bridle, J. (1987). NETspeak-a 846 re-implementation of NETtalk. Computer Speech and Language, $\mathbf{8 4 7}$ 2(3/4):289-301. $\quad \mathbf{8 4 8}$

McLachlan, G.J. and Krishnan, T. (1997). The EM Algorithm and 849 Extensions. New York, NY: John Wiley.

Moon, T.K. (1996). The expectation-maximization algorithm. IEEE 851 Signal Processing Magazine, 13(6):47-60. 852

Needleman, S.B. and Wunsch, C.D. (1970). An efficient method 853 applicable to the search for similarities in the amino acid se- $\mathbf{8 5 4}$ quences of two proteins. Journal of Molecular Biology, 48(3):444- 855 453.

Neuhoff, D.L. (1975). The Viterbi algorithm as an aid in text recog- $\mathbf{8 5 7}$ nition. In IEEE Transactions on Information Theory, IT-21:222- 858 226.

Parfitt, S.H. and Sharman, R.A. (1991). A bidirectional model of $\mathbf{8 6 0}$ English pronunciation. In Proceedings of 2nd European Confer- $\mathbf{8 6 1}$ ence on Speech Communication and Technology, Eurospeech'91. 862 Genova, Italy, Vol 2, pp. 800-804. $\quad \mathbf{8 6 3}$

Pirrelli, V. and Federici, S. (1994). On the pronunciation of unknown 864 words by analogy in text-to-speech systems. In Proceedings of the $\mathbf{8 6 5}$ 

50

Pirrelli, V. and Federici, S. (1995). You'd better say nothing than something wrong: Analogy, accuracy and text-to-speech applications. In Proceedings of 4th European Conference on Speech Communication and Technology, Eurospeech'95. Madrid, Spain, Vol 1, pp. 855-858.

Pirrelli, V. and Yvon, F. (1999). The hidden dimension: A paradigmatic view of data-driven NLP. Journal of Experimental and Theoretical Artificial Intelligence, 11(3):391-408.

Ristad, E.S. and Yianilos, P.M. (1998). Learning string-edit distance. IEEE Transactions on Pattern Analysis and Machine Intelligence, 20(5):522-532.

Sampson, G. (1985). Writing Systems. London, UK: Hutchinson.

Sejnowski, T.J. and Rosenberg, C.R. (1987). Parallel networks that learn to pronounce English text. Complex Systems, 1(1):145-168. Sullivan, K.P.H. (2001). Analogy, the corpus and pronunciation. See 2001, pp. 45-70.

Sullivan, K.P.H. and Damper, R.I. (1993). Novel-word pronunciation: A cross-language study. Speech Communication, 13(34):441-452.

Turvey, M.T., Feldman, L.B. and Lukatela, G. (1984). The SerboCroatian orthography constrains the reader to a phonologically analytic strategy. In L. Henderson (Ed.) Orthographies and Read- $\mathbf{8 8 8}$ ing: Perspectives from Cognitive Psychology, Neuropsychology $\mathbf{8 8 9}$ and Linguistics. London, UK: Lawrence Erlbaum Associates, pp. 890 81-89.

Venezky, R.L. (1965). A Study of English Spelling-to-Sound Corre- 892 spondences on Historical Principles. Ann Arbor, MI: Ann Arbor 893 Press.

Venezky, R.L. (1970). The Structure of English Orthography. The 895 Hague, The Netherlands: Mouton. $\quad 896$

Viterbi, A.J. (1967). Error bounds for convolutional codes and an $\mathbf{8 9 7}$ asymptotically optimum decoding algorithm. IEEE Transactions $\mathbf{8 9 8}$ on Information Theory, IT-13(2):260-269.

Wu, C.F.J. (1983). On the convergence properties of the EM algo- 900 rithm. Annals of Statistics, 11(1):95-103. 901

Young, S. and G. Bloothooft (Eds.) (1997). Corpus-Based Methods 902 in Language and Speech Processing. Dordrecht, The Netherlands: 903 Kluwer Academic Publishers.

904

Yvon, F. (1996a). Grapheme-to-phoneme conversion using multiple 905 unbounded overlapping chunks. In Proceedings of Conference on 906 New Methods in Natural Language Processing (NeMLaP-2'96). 907 Ankara, Turkey, pp. 218-228. 908

Yvon, F. (1996b). Prononcer par Analogie: Motivations, Formalisa- 909 tions et Évaluations. PhD thesis, ENST, Paris, France. 